\title{
Enantioselective Synthesis of Aryl Sulfoxides via Palladium-Catalyzed Arylation of Sulfenate Anions
}

Guillaume Maitro, Sophie Vogel, Mounel Sadaoui, Guillaume Prestat, David Madec,* and Giovanni Poli*

Université Pierre et Marie Curie-Paris 6, Laboratoire de Chimie Organique (UMR CNRS 7611), Institut de Chimie Moléculaire (FR 2769), Case 183, 4 Place Jussieu, F-75252, Paris Cedex 05, France

\section{Supporting Information}

\section{Table of contents}

(I) General remarks 2

(II) General procedure for palladium-catalyzed arylation of sulfenate anions 2

(III) Characterization data for aromatic sulfoxides starting from 1a 3

(IV) Characterization data for aromatic sulfoxides starting from 1b 6

(V) Characterization data for aromatic sulfoxides starting from 1c 8

(VII) Chiral SFC chromatograms 10 


\section{(I) General remarks}

Toluene was distilled from sodium/benzophenone ketyl. All other reagents and solvents were used without further purification. Ligands were obtained from Solvias AG and Strem Chemicals. Preparation of $\beta$-sulfinyl esters 1a-c and racemic sulfoxides 2-7a-c has been already described by our group. ${ }^{1} \mathrm{H}$ NMR (400 MHz) and ${ }^{13} \mathrm{C}$ NMR (100 MHz) were recorded on a Brüker ARX-400 spectrometer using the residual peak of chloroform- $d$ as internal standard. Chemical shifts are reported in ppm. IR spectra were recorded on a Brüker Tensor 27 (pike) instrument and only the strongest or structurally most important peaks were listed. Chromatographic purifications were conducted using $40-63 \mu \mathrm{m}$ silica gel and analytical TLC were performed on Merck precoated silica $60-\mathrm{F}_{254}$ plates. Enantiomeric excesses were determined by chiral SFC (Supercritical Fluid Chromatography) analysis using Chiralcel OD$\mathrm{H}$ or $\mathrm{AD}-\mathrm{H}$ column $(4.6 \mathrm{~mm} \times 250 \mathrm{~mm}, 5 \mu \mathrm{m})$. Optical rotations were measured with a Perkin-Elmer 343 Polarimeter.

\section{(II) General procedure for palladium-catalyzed arylation of sulfenate anions}

To a solution of tris(dibenzylideneacetone)dipalladium $(1 \mathrm{~mol} \%)$ in toluene $(500 \mu \mathrm{L})$ was added the appropriate ligand $(2 \mathrm{~mol} \%)$. The solution was stirred at room temperature for 5 min. Then, a solution of aryl iodide $(0.224 \mathrm{mmol}$ in $1.5 \mathrm{~mL}$ of toluene), a solution of $\beta$ sulfinylester $\left(0.187 \mathrm{mmol}\right.$ in $1.5 \mathrm{~mL}$ of toluene) and $\mathrm{Cs}_{2} \mathrm{CO}_{3}(0.748 \mathrm{mmol})$ were successively added. The resulting system was stirred vigorously at reflux for $16 \mathrm{~h}$. Then, after cooling to room temperature, $3 \mathrm{~mL}$ of a saturated aqueous $\mathrm{NH}_{4} \mathrm{Cl}$ solution were added and the aqueous phase was extracted three times with dichloromethane. The collected organic layers were dried over anhydrous $\mathrm{MgSO}_{4}$ and the solvent was removed under reduced pressure. The crude product was purified by flash chromatography.

\footnotetext{
' (a) Maitro, G.; Vogel, S.; Prestat, G.; Madec, D.; Poli, G. Org. Lett. 2006, 8, 5951-5954. (b) Maitro, G.; Prestat, G.; Madec, D.; Poli, G. J. Org. Chem. 2006, 71, 7449-7454
} 


\section{p-anisyl-p-tolyl sulfoxide 2a:}

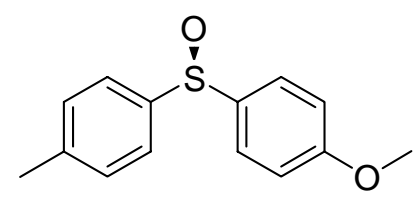

Purification by silica gel flash chromatography (cyclohexane/ethyl acetate, 9:1 $\rightarrow$ 85:15), $83 \%$ yield.

${ }^{1} \mathrm{H} \mathrm{NMR}\left(\mathrm{CDCl}_{3}, 400 \mathrm{MHz}\right): \delta$ 7.58-7.55 (m, 2H), 7.52-7.50 (m, 2H), $7.26(\mathrm{~d}, J=8.1 \mathrm{~Hz}$, 2H), 6.98-6.94 (m, 2H), $3.82(\mathrm{~s}, 3 \mathrm{H}), 2.38(\mathrm{~s}, 3 \mathrm{H})$.

${ }^{13} \mathrm{C} \mathrm{NMR}\left(\mathrm{CDCl}_{3}, 100 \mathrm{MHz}\right): \delta 161.9,142.7,141.3,137.0,130.0,127.1,124.8,114.8,55.5$, 21.4 .

IR (powder): $v$ 2921, 1592, 1493, 1248, 1036, $809 \mathrm{~cm}^{-1}$.

$\operatorname{MS}\left(\mathrm{CI} / \mathrm{NH}_{3}\right): m / z 247\left(\mathrm{MH}^{+}\right)$.

HRMS $m / z$ calculated for $\mathrm{C}_{14} \mathrm{H}_{14} \mathrm{O}_{2} \mathrm{~S}\left(\mathrm{M}^{+}\right)$: 246.0715. Found: 246.0729 .

Chiral SFC method: Chiralcel OD-H, $220 \mathrm{~nm}, 5 \mathrm{~mL} / \mathrm{min}, 5 \% \mathrm{MeOH},(R)$-isomer, $\mathrm{t}_{\mathrm{R}}=8.8$ $\min ,(S)$-isomer, $\mathrm{t}_{\mathrm{R}}=9.7 \mathrm{~min},(73 \%$ ee $)$.

$[\alpha]_{\mathrm{D}}=+16.8\left(\mathrm{c}=1.04, \mathrm{CHCl}_{3}\right)$, literature: $[\alpha]_{\mathrm{D}}=+23\left[\mathrm{c}=1.0, \mathrm{CHCl}_{3},(R)\right.$-isomer, $95 \%$ ee.$^{2}$

\section{m-anisyl-p-tolyl sulfoxide 3a:}<smiles>COc1cccc([SH](O)c2ccc(C)cc2)c1</smiles>

Purification by silica gel flash chromatography (cyclohexane/ethyl acetate, 9:1 $\rightarrow$ 85:15), 90\% yield.

${ }^{1} \mathrm{H}$ NMR $\left(\mathrm{CDCl}_{3}, 400 \mathrm{MHz}\right): \delta$ 7.56-7.54 (m, 2H), $7.35(\mathrm{t}, J=15.9 \mathrm{~Hz}, 1 \mathrm{H}), 7.26-7.24(\mathrm{~m}$, 3H), 7.17-7.14 (m, 1H), 6.97-6.94 (m, 1H), $3.84(\mathrm{~s}, 3 \mathrm{H}), 2.38(\mathrm{~s}, 3 \mathrm{H})$.

\footnotetext{
${ }^{2}$ Garcia Ruano, J. L.; Alemparte, C.; Aranda, M. T.; Zarzuelo, M. M. Org. Lett. 2003, 5, 75-78.
} 
${ }^{13} \mathrm{C} \mathrm{NMR}\left(\mathrm{CDCl}_{3}, 100 \mathrm{MHz}\right): \delta 160.4,147.2,142.5,141.8,130.3,130.1,125.1,117.3,116.9$, 109.0, 55.6, 21.5.

IR (neat): $v 2923,1593,1479,1232,1035,684 \mathrm{~cm}^{-1}$.

HRMS $m / z$ calculated for $\mathrm{C}_{14} \mathrm{H}_{14} \mathrm{O}_{2} \mathrm{~S}\left(\mathrm{M}^{+}\right)$: 246.0715. Found: 246.0729.

Chiral SFC method: Chiralcel AD-H, $220 \mathrm{~nm}, 5 \mathrm{~mL} / \mathrm{min}, 10 \% \mathrm{MeOH},(R)$-isomer, $\mathrm{t}_{\mathrm{R}}=7.7$ $\min ,(S)$-isomer, $\mathrm{t}_{\mathrm{R}}=8.4 \mathrm{~min},(68 \% \mathrm{ee})$.

$[\alpha]_{\mathrm{D}}=-31.0(\mathrm{c}=1.0, \mathrm{MeOH})$, literature: $[\alpha]_{\mathrm{D}}=+52[\mathrm{MeOH},(S)$-isomer, calculated from molecular rotation]. ${ }^{3}$

\section{o-anisyl-p-tolyl sulfoxide 4a:}

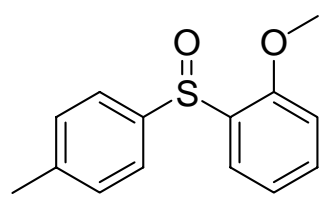

Purification by silica gel flash chromatography (cyclohexane/ethyl acetate 9:1 $\rightarrow$ 85:15), 78\% yield.

${ }^{1} \mathrm{H}$ NMR $\left(\mathrm{CDCl}_{3}, 400 \mathrm{MHz}\right): \delta 7.93(\mathrm{dd}, J=7.8,1.8 \mathrm{~Hz}, 1 \mathrm{H}), 7.60(\mathrm{~d}, J=8.1 \mathrm{~Hz}, 2 \mathrm{H}), 7.41$ (ddd, $J=8.1,7.6,1.8 \mathrm{~Hz}, 1 \mathrm{H}), 7.23(\mathrm{~d}, J=8.1 \mathrm{~Hz}, 2 \mathrm{H}), 7.17$ (ddd, $J=7.8,7.6,1.0 \mathrm{~Hz}, 1 \mathrm{H})$, $6.86(\mathrm{~d}, J=8.1 \mathrm{~Hz}, 1 \mathrm{H}), 3.81(\mathrm{~s}, 3 \mathrm{H}), 2.37(\mathrm{~s}, 3 \mathrm{H})$.

${ }^{13} \mathrm{C}$ NMR $\left(\mathrm{CDCl}_{3}, 100 \mathrm{MHz}\right): \delta 155.7,142.4,141.4,133.4,132.1,129.8,125.5,124.7,121.7$, 111.1, 55.8, 21.6.

IR (powder): $v$ 2921, 1477, 1273, 1030, $762 \mathrm{~cm}^{-1}$.

Anal. Calcd for $\mathrm{C}_{14} \mathrm{H}_{14} \mathrm{O}_{2} \mathrm{~S}: \mathrm{C}, 68.26 ; \mathrm{H}, 5.73 ; \mathrm{S}, 13.02$. Found: $\mathrm{C}, 67.86 ; \mathrm{H}, 5.77 ; \mathrm{S}, 13.32$.

Chiral SFC method: Chiralcel OD-H, $220 \mathrm{~nm}, 5 \mathrm{~mL} / \mathrm{min}, 10 \% \mathrm{MeOH}$, isomer $1, \mathrm{t}_{\mathrm{R}}=3.4 \mathrm{~min}$, isomer $2, \mathrm{t}_{\mathrm{R}}=4.5 \mathrm{~min},(0 \% \mathrm{ee})$.

\footnotetext{
${ }^{3}$ Saeva, F. D.; Rayner, D. R.; Mislow, K. J. Am. Chem. Soc. 1968, 90, 4176-4178.
} 
p-nitrophenyl-p-tolyl sulfoxide 5a:<smiles>Cc1ccc([S](O)c2ccc([N+](=O)[O-])cc2)cc1</smiles>

Purification by silica gel flash chromatography (cyclohexane/ethyl acetate, 9:1 $\rightarrow$ 85:15), $67 \%$ yield.

${ }^{1} \mathrm{H}$ NMR $\left(\mathrm{CDCl}_{3}, 400 \mathrm{MHz}\right): \delta 8.32(\mathrm{~d}, J=8.8 \mathrm{~Hz}, 2 \mathrm{H}), 7.83(\mathrm{~d}, J=9.1 \mathrm{~Hz}, 2 \mathrm{H}), 7.58(\mathrm{~d}, J=$ $8.3 \mathrm{~Hz}, 2 \mathrm{H}), 7.32$ (d, $J=7.8 \mathrm{~Hz}, 2 \mathrm{H}), 2.41(\mathrm{~s}, 3 \mathrm{H})$.

${ }^{13} \mathrm{C} \mathrm{NMR}\left(\mathrm{CDCl}_{3}, 100 \mathrm{MHz}\right): \delta 153.3,149.2,143.0,141.3,130.6,125.3,125.2,124.5,21.6$.

IR (powder): $v 3097,1527,1342,1047,851,722 \mathrm{~cm}^{-1}$.

$\operatorname{MS}\left(\mathrm{CI} / \mathrm{NH}_{3}\right): m / z 279\left(\mathrm{MNH}_{4}^{+}\right), 262\left(\mathrm{MH}^{+}\right)$.

HRMS $m / z$ calculated for $\mathrm{C}_{13} \mathrm{H}_{11} \mathrm{NO}_{3} \mathrm{~S}\left(\mathrm{M}^{+}\right): 261.0460$. Found: 261.0441 .

Chiral SFC method: Chiralcel OD-H, $220 \mathrm{~nm}, 5 \mathrm{~mL} / \mathrm{min}, 10 \% \mathrm{MeOH}$, major isomer, $\mathrm{t}_{\mathrm{R}}=5.6$ $\min$, minor isomer, $\mathrm{t}_{\mathrm{R}}=6.1 \mathrm{~min},(66 \% \mathrm{ee})$.

$[\alpha]_{\mathrm{D}}=-34.6(\mathrm{c}=1.01$, acetone $)$.

\section{p-tolyl-p-trifluoromethylphenyl sulfoxide 6a:}<smiles>Cc1ccc([S](O)c2ccc(C(F)(F)F)cc2)cc1</smiles>

Purification by silica gel flash chromatography (cyclohexane/ ethyl acetate, 9:1 $\rightarrow$ 85:15) (98\% yield).

${ }^{1} \mathrm{H}$ NMR $\left(\mathrm{CDCl}_{3}, 400 \mathrm{MHz}\right): \delta 7.77(\mathrm{~d}, J=8.6 \mathrm{~Hz}, 2 \mathrm{H}), 7.71(\mathrm{~d}, J=8.4 \mathrm{~Hz}, 2 \mathrm{H}), 7.56(\mathrm{~d}, J=$ $8.4 \mathrm{~Hz}, 2 \mathrm{H}), 7.29$ (d, $J=7.8 \mathrm{~Hz}, 2 \mathrm{H}), 2.38$ (s, 3H).

${ }^{13} \mathrm{C} \mathrm{NMR}\left(\mathrm{CDCl}_{3}, 100 \mathrm{MHz}\right): \delta 150.3,142.5,141.9,132.7$ (q, $\left.J=32 \mathrm{~Hz}\right), 130.4,126.3$, $125.2,124.9,123.6(\mathrm{q}, J=271 \mathrm{~Hz}), 21.5$

IR (neat): $v 2924,1321,1127,1056,808,698 \mathrm{~cm}^{-1}$. 
HRMS $m / z$ calculated for $\mathrm{C}_{14} \mathrm{H}_{11} \mathrm{OF}_{3} \mathrm{~S}\left(\mathrm{M}^{+}\right)$: 284.0483. Found: 284.0463.

Chiral SFC method: Chiralcel OD-H, $220 \mathrm{~nm}, 5 \mathrm{~mL} / \mathrm{min}, 2.5 \% \mathrm{MeOH},(R)$-isomer, $\mathrm{t}_{\mathrm{R}}=5.4$ $\min ,(S)$-isomer, $\mathrm{t}_{\mathrm{R}}=5.8 \mathrm{~min},(80 \% \mathrm{ee})$.

$[\alpha]_{\mathrm{D}}=-36.0(\mathrm{c}=1.00, \mathrm{EtOH})$, literature: $[\alpha]_{\mathrm{D}}=+57\left[\mathrm{EtOH},(S)\right.$-isomer, $100 \%$ ee.$^{4}$

\section{2-thiophenyl-p-tolyl sulfoxide 7a:}<smiles>Cc1ccc([S](O)c2cccs2)cc1</smiles>

Purification by silica gel flash chromatography (cyclohexane/ethyl acetate, 9:1), 85\% yield.

${ }^{1} \mathrm{H}$ NMR $\left(\mathrm{CDCl}_{3}, 400 \mathrm{MHz}\right): \delta 7.61-7.55(\mathrm{~m}, 4 \mathrm{H}), 7.32(\mathrm{~d}, J=8.1 \mathrm{~Hz}, 2 \mathrm{H}), 7.06(\mathrm{dd}, J=5.1$, $3.8 \mathrm{~Hz}, 1 \mathrm{H}), 2.42$ (s, 3H).

${ }^{13} \mathrm{C} \mathrm{NMR}\left(\mathrm{CDCl}_{3}, 100 \mathrm{MHz}\right): \delta 148.5,142.0,141.9,132.3,131.2,130.0,127.4,124.5,21.6$.

IR (neat): $v 2921,1402,1082,1042,808,712 \mathrm{~cm}^{-1}$.

HRMS $m / z$ calculated for $\mathrm{C}_{11} \mathrm{H}_{10} \mathrm{OS}_{2}\left(\mathrm{M}^{+}\right): 222.0173$. Found: 222.0183.

$[\alpha]_{\mathrm{D}}=-54.4(\mathrm{c}=1.02$, acetone $)$, literature: $[\alpha]_{\mathrm{D}}=+110[\mathrm{c}=2.5$, acetone, $(S)$-isomer, $100 \%$ ee]. ${ }^{5}$

2-naphthyl-p-anisyl sulfoxide $2 b$ :<smiles>COc1ccc([S](O)c2ccc3ccccc3c2)cc1</smiles>

\footnotetext{
${ }^{4}$ Rayner, D. R.; Gordon, A. J.; Mislow, K. J. Am. Chem. Soc. 1968, 90, 4854-4860.

${ }^{5}$ Girodier, L.; Maignan, C.; Rouessac, F. Tetrahedron: Asymmetry 1992, 3, 857-858.
} 
Purification by silica gel flash chromatography (cyclohexane/ethyl acetate, 9:1 $\rightarrow$ 7:3), 75\% yield.

${ }^{1} \mathrm{H}$ NMR $\left(\mathrm{CDCl}_{3}, 400 \mathrm{MHz}\right): \delta 8.33(\mathrm{~s}, 1 \mathrm{H}), 7.98-7.95(\mathrm{~m}, 1 \mathrm{H}), 7.88-7.85(\mathrm{~m}, 2 \mathrm{H}), 7.64-7.57$ (m, 4H), 7.46-7.43 (m, 1H), $6.96(\mathrm{~d}, J=8.8 \mathrm{~Hz}, 2 \mathrm{H}), 3.82$ (s, 3H).

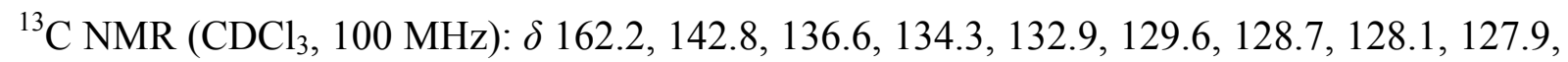
127.6, 127.3, 125.0, 120.8, 115.0, 55.6.

IR (powder): $v$ 2964, 1592, 1494, 1252, 1039, $816 \mathrm{~cm}^{-1}$.

$\operatorname{MS}\left(\mathrm{CI} / \mathrm{NH}_{3}\right): m / z 300\left(\mathrm{MNH}_{4}{ }^{+}\right), 283\left(\mathrm{M}^{+}\right)$.

HRMS $m / z$ calculated for $\mathrm{C}_{17} \mathrm{H}_{14} \mathrm{O}_{2} \mathrm{~S}\left(\mathrm{M}^{+}\right)$: 282.0715. Found: 282.0719 .

Chiral SFC method: Chiralcel OD-H, $220 \mathrm{~nm}, 5 \mathrm{~mL} / \mathrm{min}, 20 \% \mathrm{MeOH}$, minor isomer, $\mathrm{t}_{\mathrm{R}}=3.7$ $\min$, major isomer, $\mathrm{t}_{\mathrm{R}}=4.3 \mathrm{~min},(72 \% \mathrm{ee})$.

$[\alpha]_{\mathrm{D}}=+48.5(\mathrm{c}=1.02$, acetone $)$.

\section{2-naphthyl-p-tolyl sulfoxide $3 b$ :}<smiles>Cc1ccc([S](O)c2ccc3ccccc3c2)cc1</smiles>

Purification by silica gel flash chromatography (cyclohexane/ethyl acetate, 9:1 $\rightarrow$ 8:2), 93\% yield.

${ }^{1} \mathrm{H}$ NMR $\left(\mathrm{CDCl}_{3}, 400 \mathrm{MHz}\right): \delta 8.33-8.32(\mathrm{~m}, 1 \mathrm{H}), 7.97-7.94(\mathrm{~m}, 1 \mathrm{H}), 7.88-7.83(\mathrm{~m}, 2 \mathrm{H})$, 7.60-7.56 (m, 4H), $7.48(\mathrm{dd}, J=8.6,1.8 \mathrm{~Hz}, 1 \mathrm{H}), 7.26$ (d, $J=8.1 \mathrm{~Hz}, 2 \mathrm{H}), 2.36(\mathrm{~s}, 3 \mathrm{H})$.

${ }^{13} \mathrm{C} \mathrm{NMR}\left(\mathrm{CDCl}_{3}, 100 \mathrm{MHz}\right): \delta$ 142.8, 142.3, 141.8, 134.4, 132.9, 130.1, 129.7, 128.7, 128.1, 127.9, 127.3, 125.3, 125.2, 120.8, 21.50.

Chiral SFC method: Chiralcel OD-H, $5 \mathrm{~mL} / \mathrm{min}, 20 \% \mathrm{MeOH},(R)$-isomer, $\mathrm{t}_{\mathrm{R}}=3.0 \mathrm{~min},(S)$ isomer, $\mathrm{t}_{\mathrm{R}}=3.5 \mathrm{~min},(65 \% \mathrm{ee})$.

$[\alpha]_{\mathrm{D}}=+28.0\left(\mathrm{c}=1.04, \mathrm{CHCl}_{3}\right)$, literature: $[\alpha]_{\mathrm{D}}=+47\left[\mathrm{CHCl}_{3},(S)\right.$-isomer, $100 \%$ ee.$^{6}$

${ }^{6}$ Mislow, K.; Axelrod, M.; Rayner, D. R.; Gotthardt, H.; Coyne, L. M.; Hammond, G. S. J. Am. Chem. Soc. 1965, 87, 4958-4959. 


\section{(VI) Characterization data for aromatic sulfoxides starting from 1c}

\section{p-anisyl-benzyl sulfoxide 2c:}

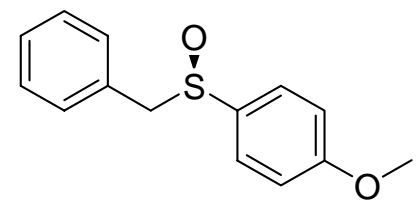

Purification by silica gel flash chromatography (cyclohexane/ethyl acetate, 95:5 $\rightarrow$ 7:3), 67\% yield.

${ }^{1} \mathrm{H}$ NMR $\left(\mathrm{CDCl}_{3}, 400 \mathrm{MHz}\right): \delta$ 7.33-7.24 (m, 5H), 7.01-6.92 (m, 4H), $4.04(\mathrm{AB}, J=12.4 \mathrm{~Hz}$, $2 \mathrm{H}), 3.85(\mathrm{~s}, 3 \mathrm{H})$.

${ }^{13} \mathrm{C} \mathrm{NMR}\left(\mathrm{CDCl}_{3}, 100 \mathrm{MHz}\right): \delta 162.1,133.7,130.5,129.4,128.6,128.3,126.5,114.4,63.8$, 55.6 .

Chiral SFC method: Chiralcel OD-H, $5 \mathrm{~mL} / \mathrm{min}, 5 \% \mathrm{MeOH},(S)$-isomer, $\mathrm{t}_{\mathrm{R}}=9.5 \mathrm{~min},(R)-$ isomer, $\mathrm{t}_{\mathrm{R}}=10.2 \mathrm{~min},(40 \% \mathrm{ee})$.

$[\alpha]_{\mathrm{D}}=+72.6(\mathrm{c}=1.00$, acetone $)$, literature: $[\alpha]_{\mathrm{D}}=-47.1[\mathrm{c}=1.03$, acetone, $(S)$-isomer, 92\% ee]. ${ }^{7}$

\section{benzyl-p-tolyl sulfoxide 3c:}

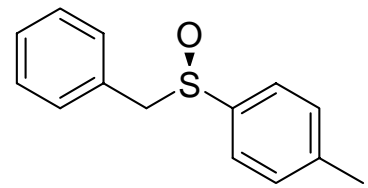

Purification by silica gel flash chromatography (cyclohexane/ethyl acetate, 9:1 $\rightarrow$ 7:3), 71\% yield.

\footnotetext{
${ }^{7}$ Donnoli, M. I.; Superchi, S.; Rosini, C. J. Org. Chem. 1998, 63, 9392-9395.
} 
${ }^{1} \mathrm{H}$ NMR $\left(\mathrm{CDCl}_{3}, 400 \mathrm{MHz}\right): \delta 7.33-7.23(\mathrm{~m}, 7 \mathrm{H}), 7.02-6.99(\mathrm{~m}, 2 \mathrm{H}), 4.05(\mathrm{AB}, J=12.4 \mathrm{~Hz}$, $2 \mathrm{H}), 2.41(\mathrm{~s}, 3 \mathrm{H})$.

${ }^{13} \mathrm{C} \mathrm{NMR}\left(\mathrm{CDCl}_{3}, 100 \mathrm{MHz}\right): \delta 142.8,141.7,130.4,129.6,128.9,128.5,128.3,124.5,63.8$, 21.5.

Chiral SFC method: Chiralcel AD-H, $5 \mathrm{~mL} / \mathrm{min}, 20 \% \mathrm{MeOH},(S)$-isomer, $\mathrm{t}_{\mathrm{R}}=2.3 \mathrm{~min},(R)-$ isomer, $\mathrm{t}_{\mathrm{R}}=2.5 \mathrm{~min},(47 \% \mathrm{ee})$.

$[\alpha]_{\mathrm{D}}=+99.5(\mathrm{c}=0.71$, acetone $)$, literature: $[\alpha]_{\mathrm{D}}=-179.1[\mathrm{c}=1.3$, acetone, $(S)$-isomer, $94 \%$ ee]. ${ }^{8}$

${ }^{8}$ Kelly, P.; Lawrence, S. E.; Maguire, A. R. Eur. J. Org. Chem. 2006, 4500-4509. 
CCP25F.tmp.DAT - Detector 1 Signal (UV)

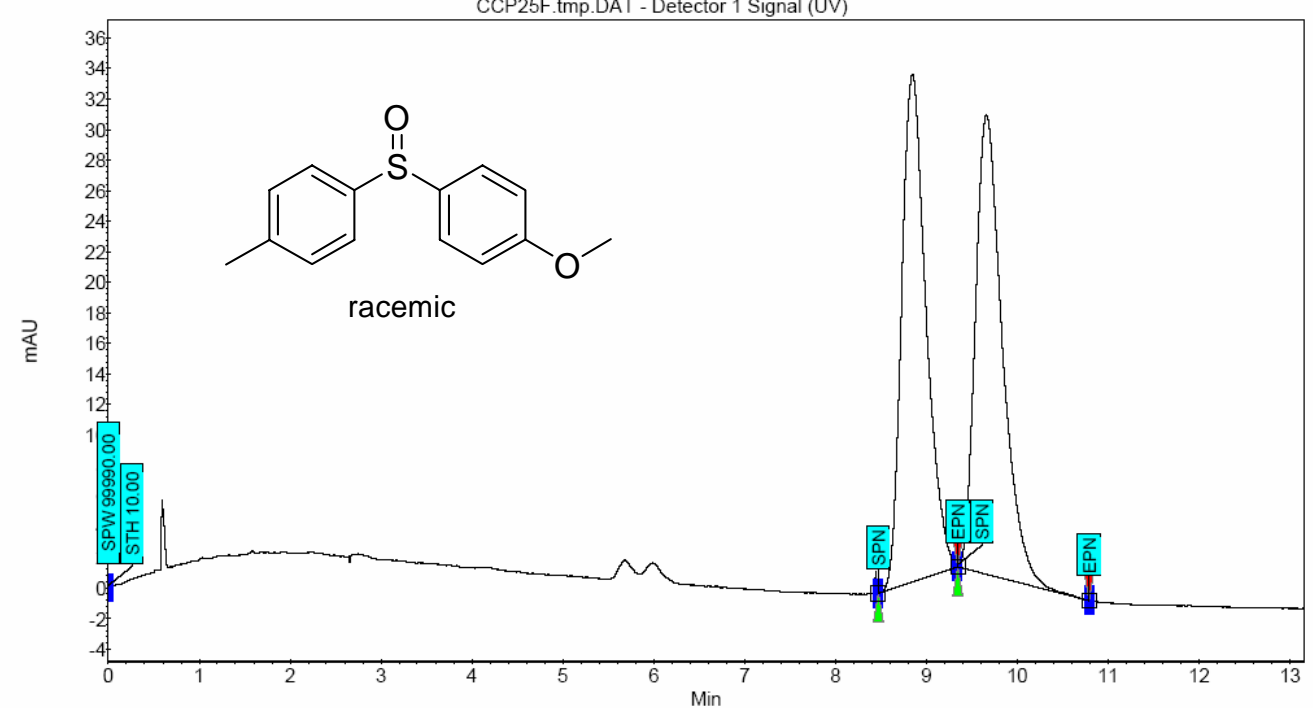

\begin{tabular}{|c|r|r|r|r|r|r|r|}
\hline \multicolumn{1}{|l|}{ Index } & Name & \multicolumn{1}{c}{ Time Height } & \multicolumn{1}{|c|}{ Area } & Area & Selectivity & Res. HW \\
\hline \hline & & {$[$ Min] } & {$[\mu \mathrm{V}]$} & {$[\mu \mathrm{V} . \mathrm{Min}]$} & {$[\%]$} & & \\
\hline 1 & UNKNOWN & 8.84 & 33.3 & 10.3 & 50.016 & 0.00 & 0.00 \\
\hline 2 & UNKNOWN & 9.66 & 30.1 & 10.3 & 49.984 & 1.09 & 1.62 \\
\hline & & & & & & & \\
\hline Total & & & 63.4 & 20.6 & 100.000 & & \\
\hline
\end{tabular}

MS24dmo

MS24dmo

sulfoxyde colonne OD-H $5 \mathrm{~m} / \mathrm{min}, 5 \% \mathrm{MeOH} 220 \mathrm{~nm}$

4/6/2007 2:15:05 PM

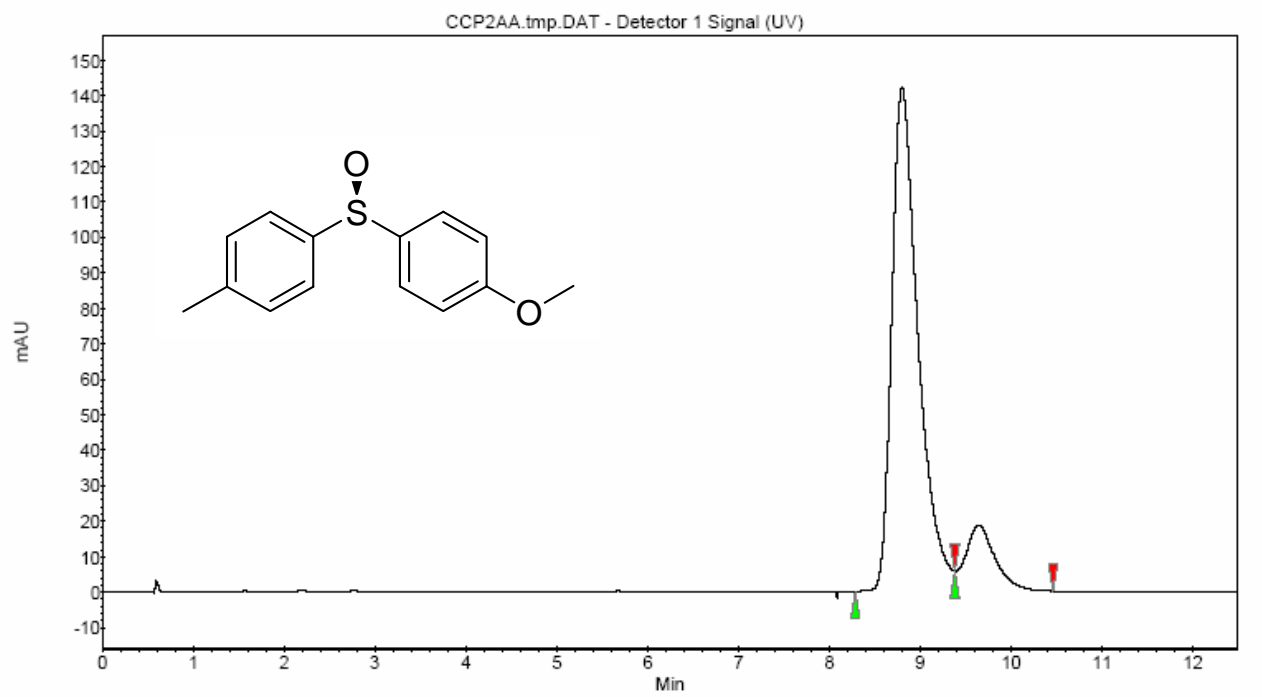

\begin{tabular}{|c|r|r|r|r|r|r|r|}
\hline \multicolumn{2}{|c|}{ Index Name } & \multicolumn{1}{c|}{ Time Height } & Area & \multicolumn{3}{|c|}{ Area } & \multicolumn{2}{c|}{ Selectivity Res. HW } \\
\hline \hline & & {$[$ Min] } & {$[\mu \mathrm{V}]$} & {$[\mu \mathrm{V} . \mathrm{Min}]$} & {$[\%]$} & & \\
\hline 2 & UNKNOWN & 8.79 & 137.1 & 41.9 & 91.389 & 0.00 & 0.00 \\
\hline 1 & UNKNOWN & 9.64 & 14.3 & 3.9 & 8.611 & 1.10 & 1.78 \\
\hline & & & & & & & \\
\hline Total & & & 151.4 & 45.8 & 100.000 & & \\
\hline
\end{tabular}


MS31rac 100

ESPCI - Laboratoire de Chimie Organique

sulfoxyde colonne AD-H $5 \mathrm{ml} / \mathrm{min}, 10 \% \mathrm{MeOH} 220 \mathrm{~nm}$

5/31/2007 4:24:17 PM

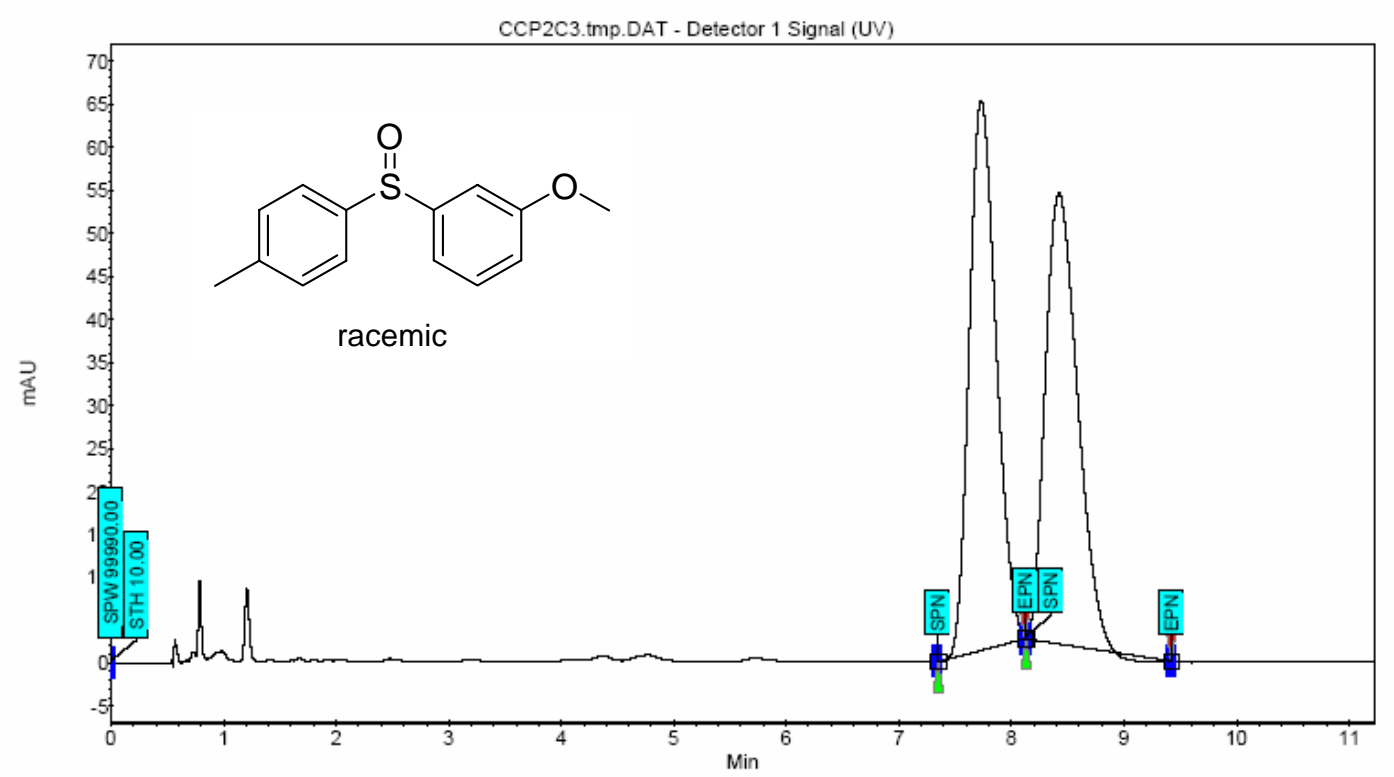

\begin{tabular}{|c|l|r|r|r|r|r|r|}
\hline \multicolumn{1}{|l|}{ Index } & Name & \multicolumn{1}{l|}{ Time Height } & Area & \multicolumn{2}{|r|}{ Area } & \multicolumn{2}{r|}{ Selectivity Res. HW } \\
\hline \hline & & {$[\mathrm{Min}]$} & {$[\mu \mathrm{V}]$} & {$[\mu \mathrm{V} . \mathrm{Min}]$} & {$[\%]$} & & \\
\hline 1 & UNKNOWN & 7.73 & 64.0 & 17.7 & 50.687 & 0.00 & 0.00 \\
\hline 2 & UNKNOWN & 8.42 & 52.4 & 17.2 & 49.313 & 1.09 & 1.41 \\
\hline & & & & & & & \\
\hline Total & & & 116.4 & 35.0 & 100.000 & & \\
\hline
\end{tabular}

MS31ee0

MS31ee0

$\mathrm{ESPCl}$ - Laboratoire de Chimie Organique

sulfoxyde colonne AD-H $5 \mathrm{ml} / \mathrm{min}, 10 \% \mathrm{MeOH} 220 \mathrm{~nm}$

5/31/2007 4:16:49 PM

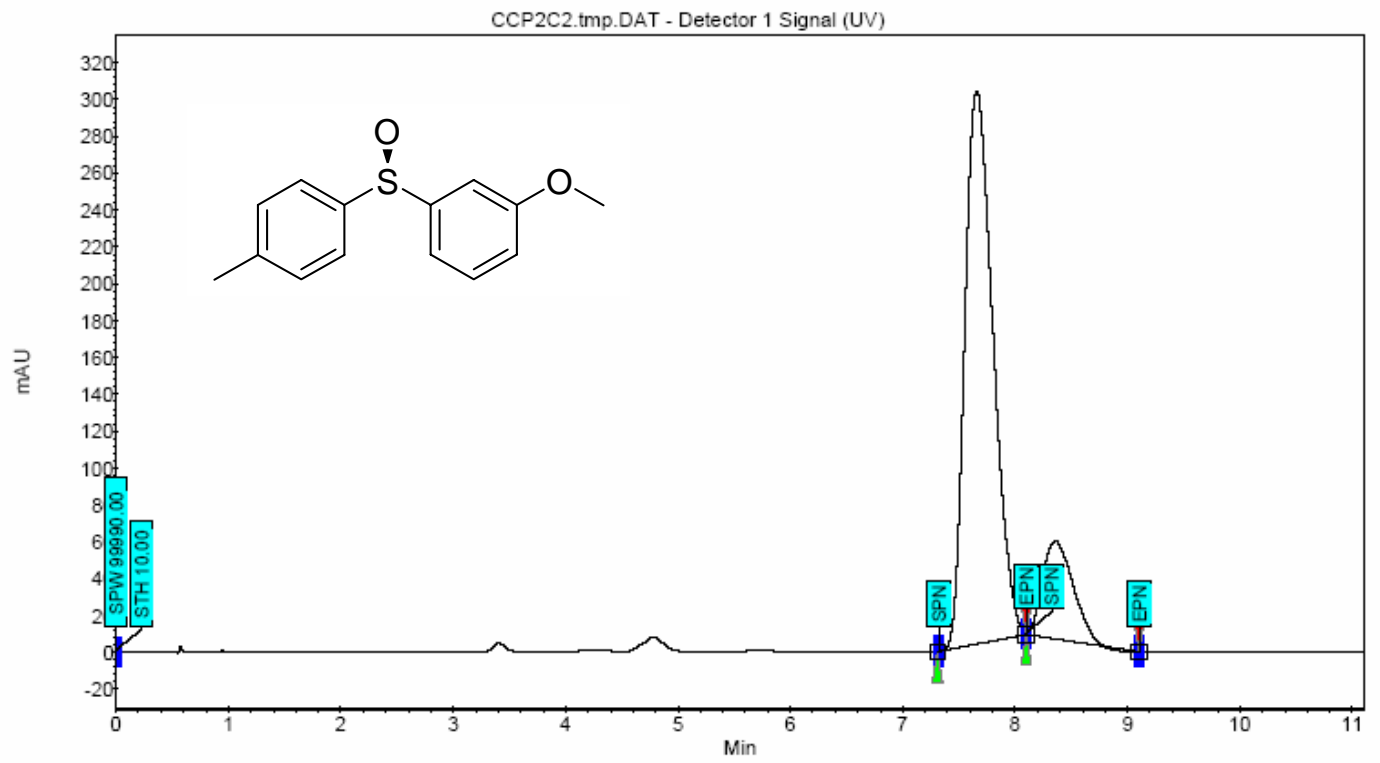

\begin{tabular}{|c|l|r|r|r|r|r|r|}
\hline \multicolumn{1}{|l|}{ Index } & Name & \multicolumn{1}{l}{ Time } & Height & Area & \multicolumn{2}{|c|}{ Area Selectivity Res. HW } \\
\hline \hline & & {$[\mathrm{Min}]$} & {$[\mu \mathrm{V}]$} & {$[\mu \mathrm{V} . \mathrm{Min}]$} & {$[\%]$} & & \\
\hline 1 & UNKNOWN & 7.66 & 300.4 & 87.1 & 83.946 & 0.00 & 0.00 \\
\hline 2 & UNKNOWN & 8.36 & 53.1 & 16.6 & 16.054 & 1.09 & 1.43 \\
\hline & & & & & & & \\
\hline Total & & & 353.5 & 103.7 & 100.000 & & \\
\hline
\end{tabular}

11 
sulfoxyde colonne OD-H $5 \mathrm{ml} / \mathrm{min}, 10 \% \mathrm{MeOH} 220 \mathrm{~nm}$

4/6/2007 1:55:58 PM

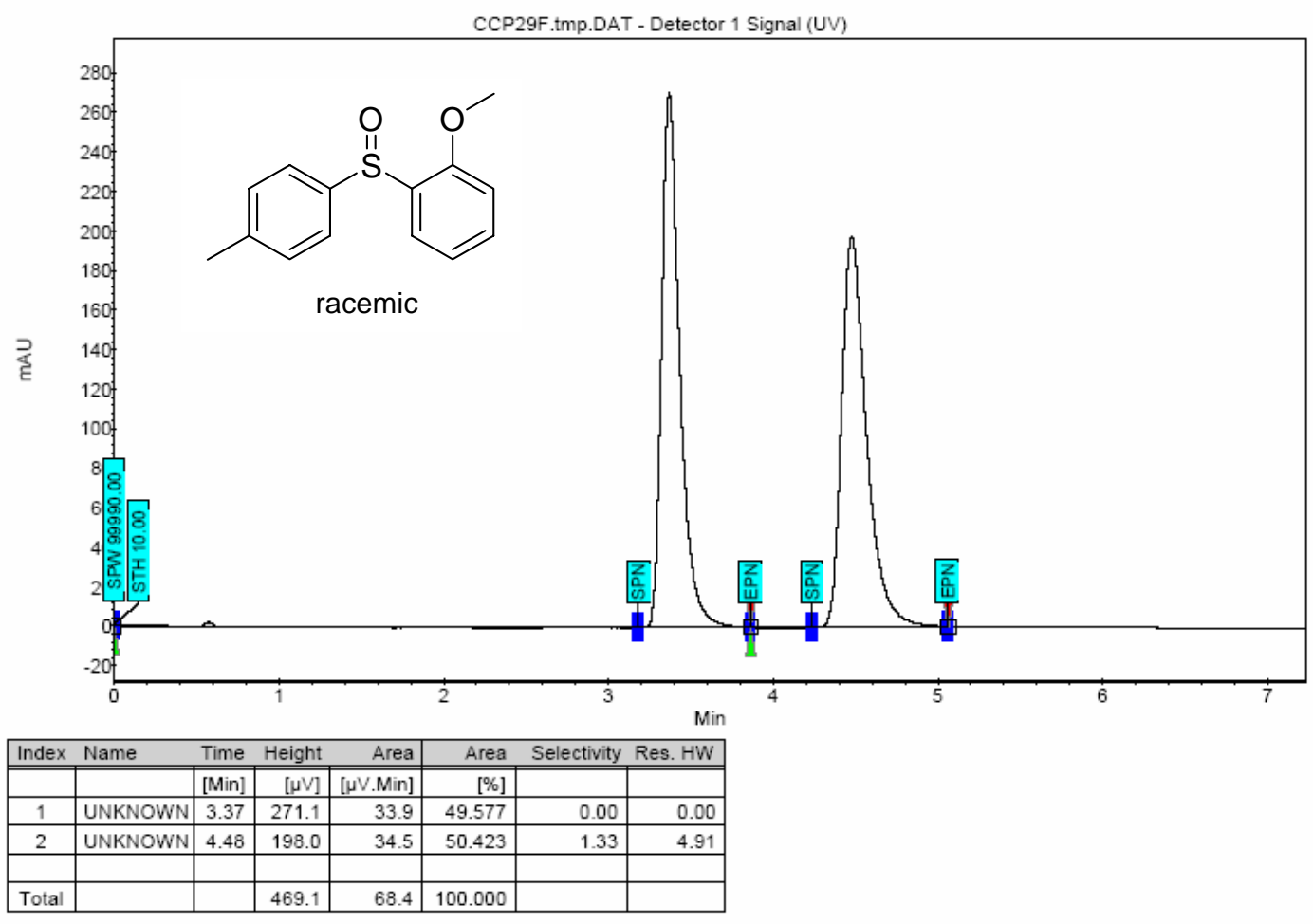

MS29ee0

ESPCI - Laboratoire de Chimie Organique

MS29ee0

sulfoxyde colonne OD-H $5 \mathrm{ml} / \mathrm{min}, 10 \% \mathrm{MeOH} 220 \mathrm{~nm}$

4/6/2007 1:57:20 PM

CCP2A0.tmp.DAT - Detector 1 Signal (UV)

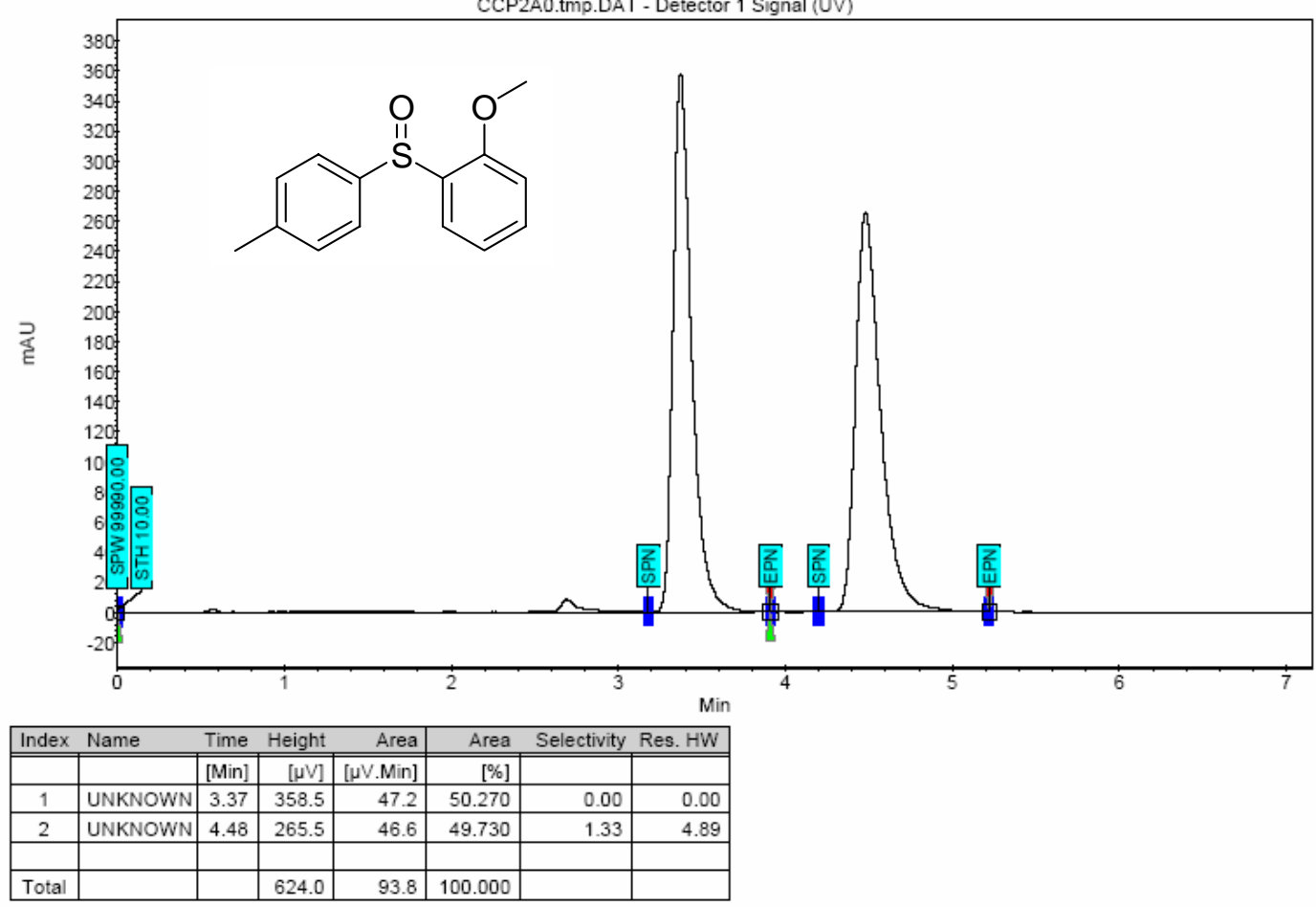


sulfoxyde colonne OD-H $5 \mathrm{~m} / / \mathrm{min}, 10 \% \mathrm{MeOH} 220 \mathrm{~nm}$

5/31/2007 4:44:40 PM

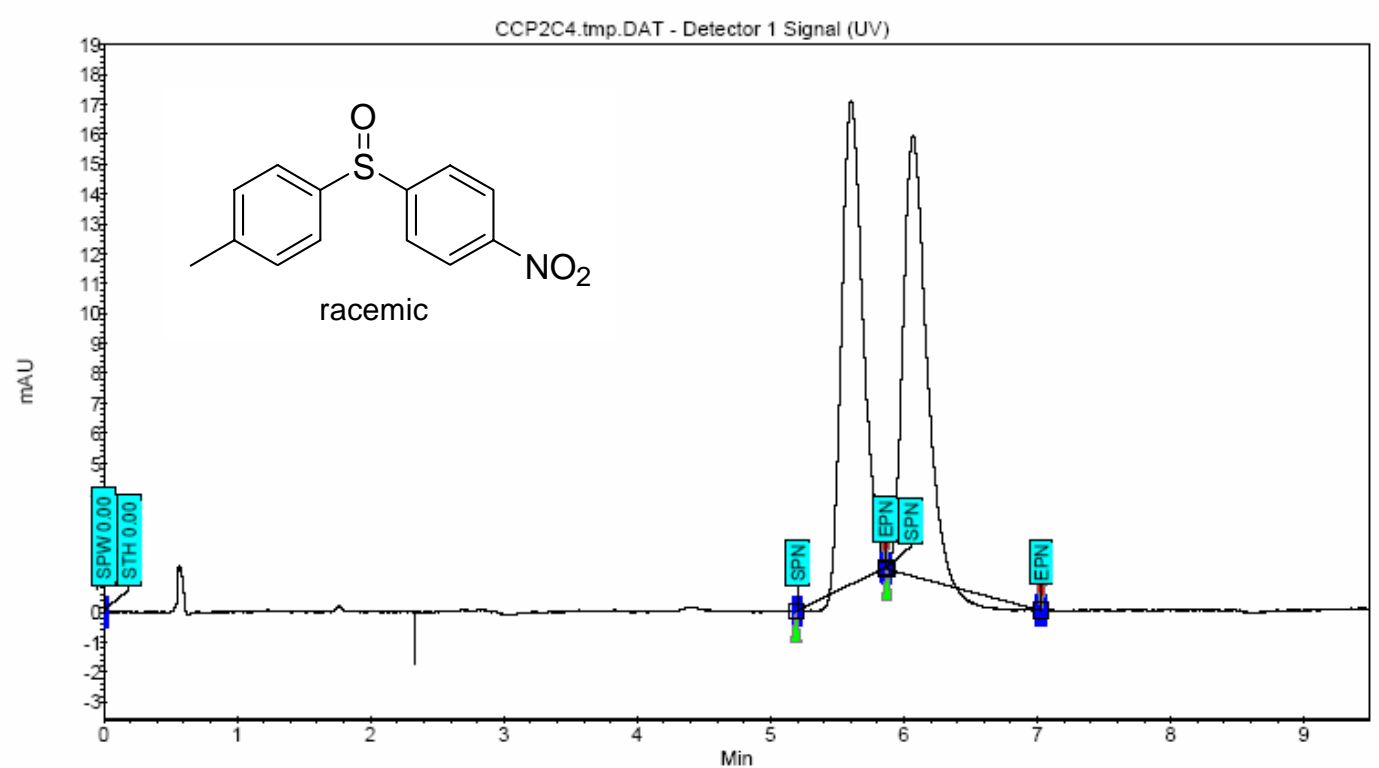

\begin{tabular}{|c|l|r|r|r|r|r|r|}
\hline \multicolumn{2}{|l|}{ Index Name } & \multicolumn{1}{l|}{ Time Height } & Area & \multicolumn{2}{|c|}{ Area } & \multicolumn{2}{r|}{ Selectivity Res. HW } \\
\hline \hline & & {$[\mathrm{Min}]$} & {$[\mu \mathrm{V}]$} & {$[\mu \mathrm{\mu} . \mathrm{Min}]$} & {$[\%]$} & & \\
\hline 1 & UNKNOWN & 5.60 & 16.2 & 2.9 & 50.767 & 0.00 & 0.00 \\
\hline 2 & UNKNOWN & 6.07 & 14.8 & 2.8 & 49.233 & 1.08 & 1.54 \\
\hline & & & & & & & \\
\hline Total & & & 31.0 & 5.7 & 100.000 & & \\
\hline
\end{tabular}

\section{MS35ee0}

MS35ee0

ESPCI - Laboratoire de Chimie Organique

sulfoxyde colonne OD-H $5 \mathrm{ml} / \mathrm{min}, 10 \% \mathrm{MeOH} 220 \mathrm{~nm}$

5/31/2007 4:07:45 PM

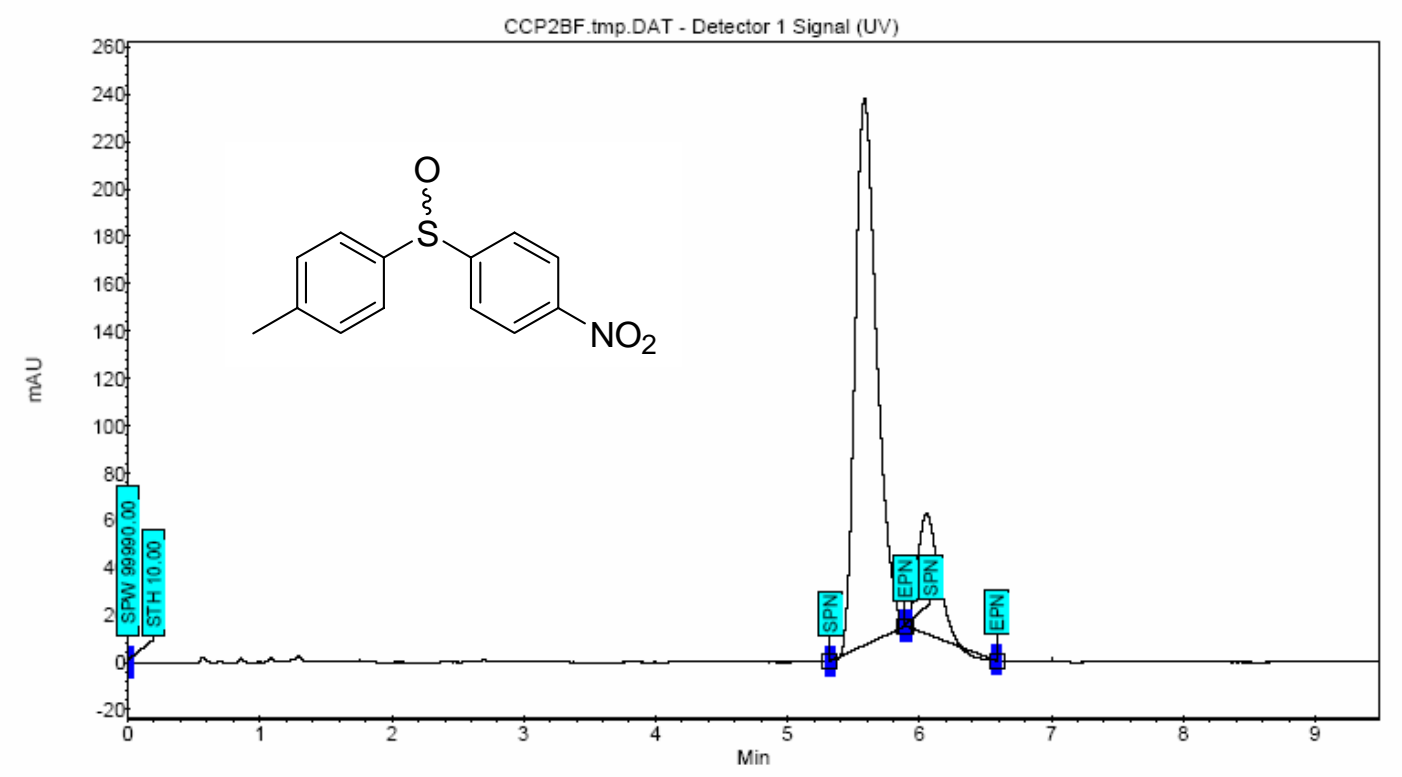

\begin{tabular}{|c|r|r|r|r|r|r|r|}
\hline Index & Name & \multicolumn{1}{l|}{ Time Height } & Area & \multicolumn{2}{|c|}{ Area Selectivity Res. HW } \\
\hline \hline & & {$[$ Min] } & {$[\mu \mathrm{V}]$} & {$[\mu \mathrm{V} . \mathrm{Min}]$} & {$[\%]$} & & \\
\hline 1 & UNKNOWN & 5.58 & 231.4 & 44.3 & 83.183 & 0.00 & 0.00 \\
\hline 2 & UNKNOWN & 6.05 & 50.5 & 9.0 & 16.817 & 1.08 & 1.59 \\
\hline & & & & & & & \\
\hline Total & & & 281.9 & 53.3 & 100.000 & & \\
\hline
\end{tabular}


MS 28rac0

ESPCl - Laboratoire de Chime Organique

MS28rac

$220 \mathrm{~nm}$

$5 / 31 / 2007$ clone OD-

CCP2C5.tmp.DAT - Detector 1 Signal (UV)

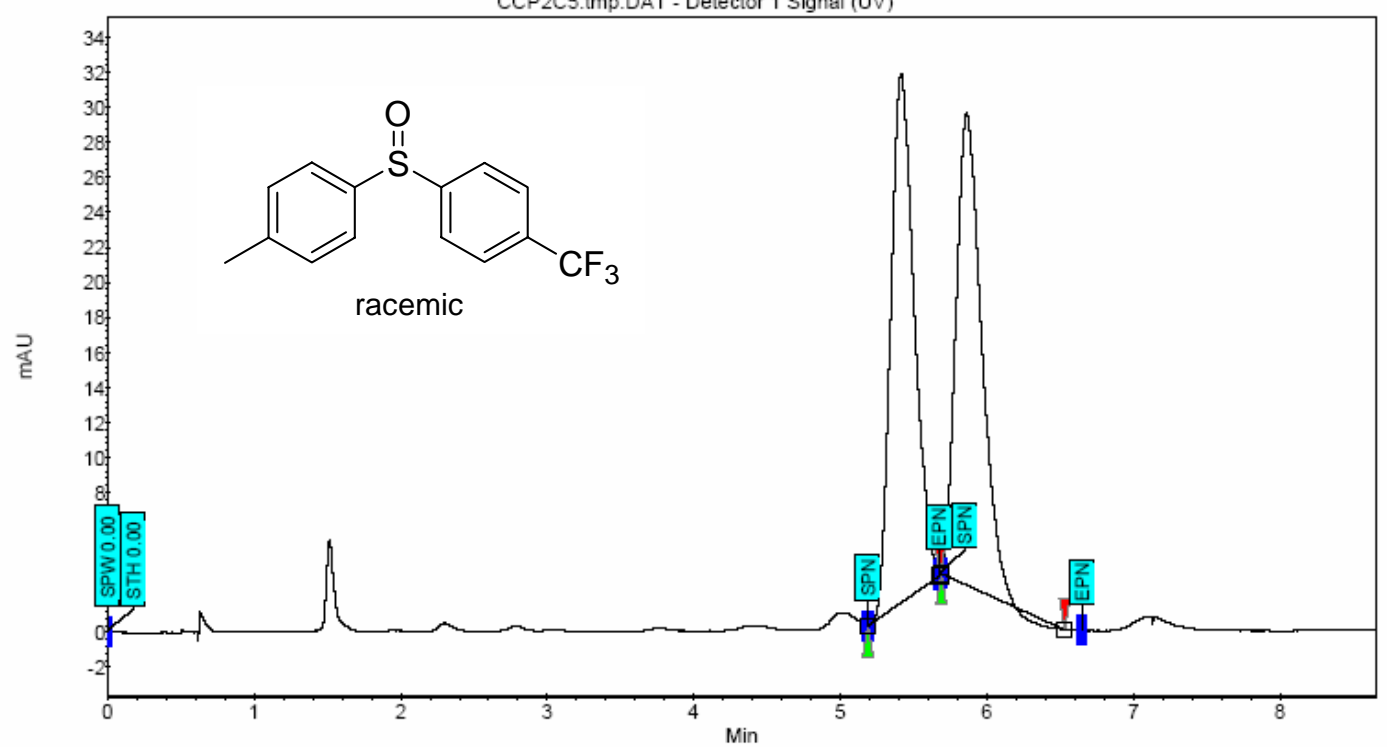

\begin{tabular}{|c|l|r|r|r|r|r|r|}
\hline \multicolumn{2}{|l|}{ Index Name } & \multicolumn{1}{l|}{ Time Height } & Area & \multicolumn{2}{|c|}{ Area } & \multicolumn{2}{r|}{ Selectivity Res. HW } \\
\hline \hline & & {$[$ Min] } & {$[\mu \mathrm{V}]$} & {$[\mu \mathrm{V}$. Min] } & {$[\%]$} & & \\
\hline 1 & UNKNOWN & 5.41 & 30.3 & 5.6 & 51.074 & 0.00 & 0.00 \\
\hline 2 & UNKNOWN & 5.86 & 27.0 & 5.4 & 48.926 & 1.08 & 1.47 \\
\hline & & & & & & & \\
\hline Total & & & 57.3 & 11.0 & 100.000 & & \\
\hline
\end{tabular}

MS 28ee0

ESPCl - Laboratoire de Chime Organique

MS28ee0

sulfoxyde colone OD -H $5 \mathrm{ml} / \mathrm{min}, 2.5 \% \mathrm{MeOH} 220 \mathrm{~nm}$

5/31/2007 4:58:19 PM

CCP2C6.tmp.DAT - Detector 1 Signal (UV)

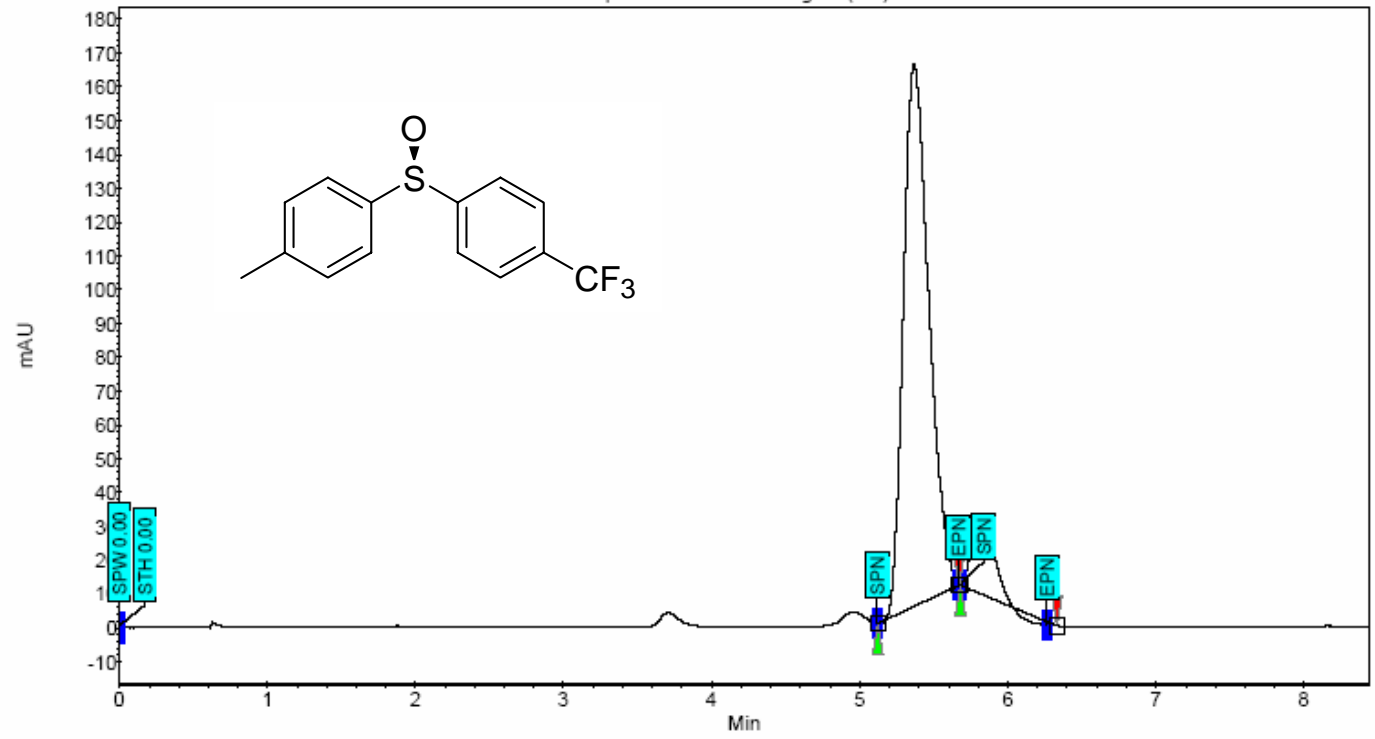

\begin{tabular}{|c|l|r|r|r|r|r|r|}
\hline \multicolumn{2}{|l|}{ Index Name } & \multicolumn{1}{l|}{ Time Height } & Area & \multicolumn{2}{|c|}{ Area } & \multicolumn{2}{r|}{ Selectivity Res. HW } \\
\hline \hline & & {$[$ Min] } & {$[\mu \mathrm{V}]$} & {$[\mu \vee . M i n]$} & {$[\%]$} & & \\
\hline 1 & UNKNOWN & 5.37 & 160.8 & 32.1 & 89.901 & 0.00 & 0.00 \\
\hline 2 & UNKNOWN & 5.82 & 22.7 & 3.6 & 10.099 & 1.09 & 1.52 \\
\hline & & & & & & & \\
\hline Total & & & 183.6 & 35.7 & 100.000 & & \\
\hline
\end{tabular}

14 
sulfoxyde colonne OD-H $5 \mathrm{ml} / \mathrm{min}, 20 \% \mathrm{MeOH} 220 \mathrm{~nm}$

4/6/2007 12:21:07 PM

CCP273.tmp.DAT - Detector 1 Signal (UV)

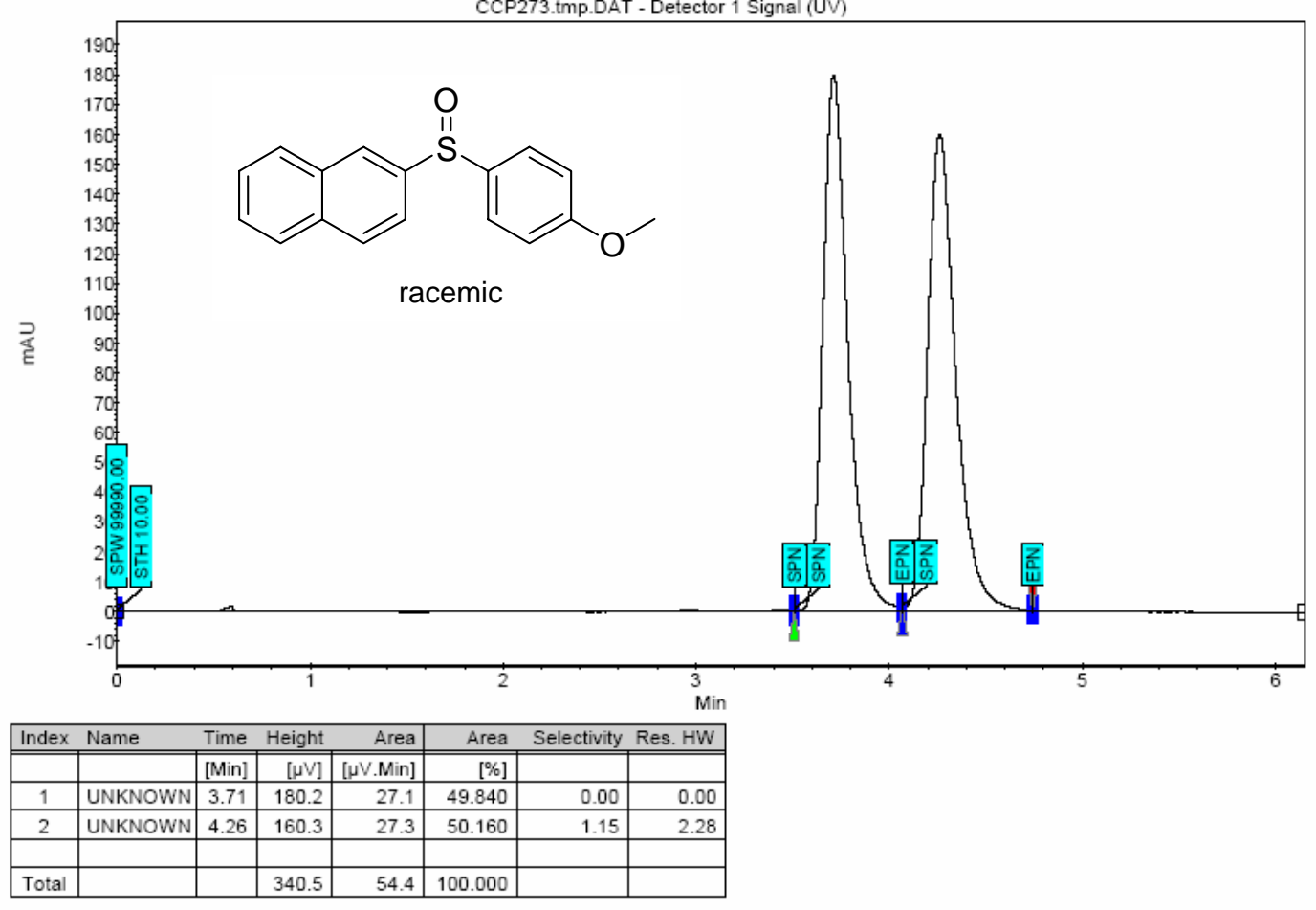

MS 33 eed

ESPCI - Laboratoire de Chimie Organique

MS33ee0

sulfoxyde colonne OD-H $5 \mathrm{ml} / \mathrm{min}, 20 \% \mathrm{MeOH} 220 \mathrm{~nm}$

4/6/2007 12:35:49 PM

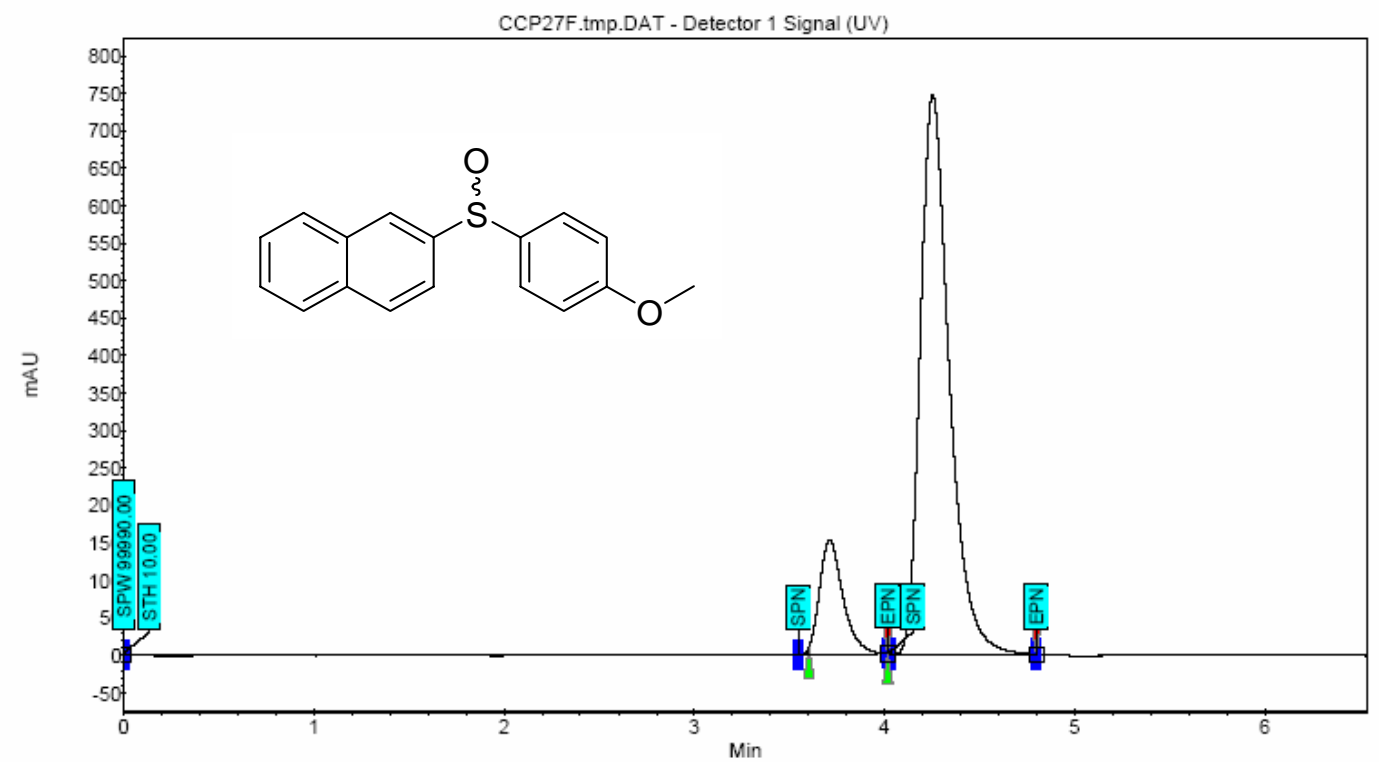

\begin{tabular}{|c|l|r|r|r|r|r|r|}
\hline \multicolumn{1}{|l|}{ Index Name } & \multicolumn{1}{c|}{ Time Height } & Area & \multicolumn{2}{|c|}{ Area } & \multicolumn{2}{l|}{ Selectivity Res. HW } \\
\hline \hline & & {$[\mathrm{Min}]$} & {$[\mu \mathrm{V}]$} & {$[\mu \mathrm{V} . \mathrm{Min}]$} & {$[\%]$} & & \\
\hline 2 & UNKNOWN & 3.71 & 152.3 & 21.7 & 14.239 & 0.00 & 0.00 \\
\hline 1 & UNKNOWN & 4.25 & 747.6 & 130.6 & 85.761 & 1.15 & 2.24 \\
\hline & & & & & & & \\
\hline Total & & & 899.9 & 152.3 & 100.000 & & \\
\hline
\end{tabular}


MS 34 rac0

MS34rac

ESPCI - Laboratoire de Chimie Organique

sulfoxyde colonne OD-H $5 \mathrm{~m} / \mathrm{min}, 20 \% \mathrm{MeOH} 220 \mathrm{~nm}$

4/6/2007 12:58:25 PM

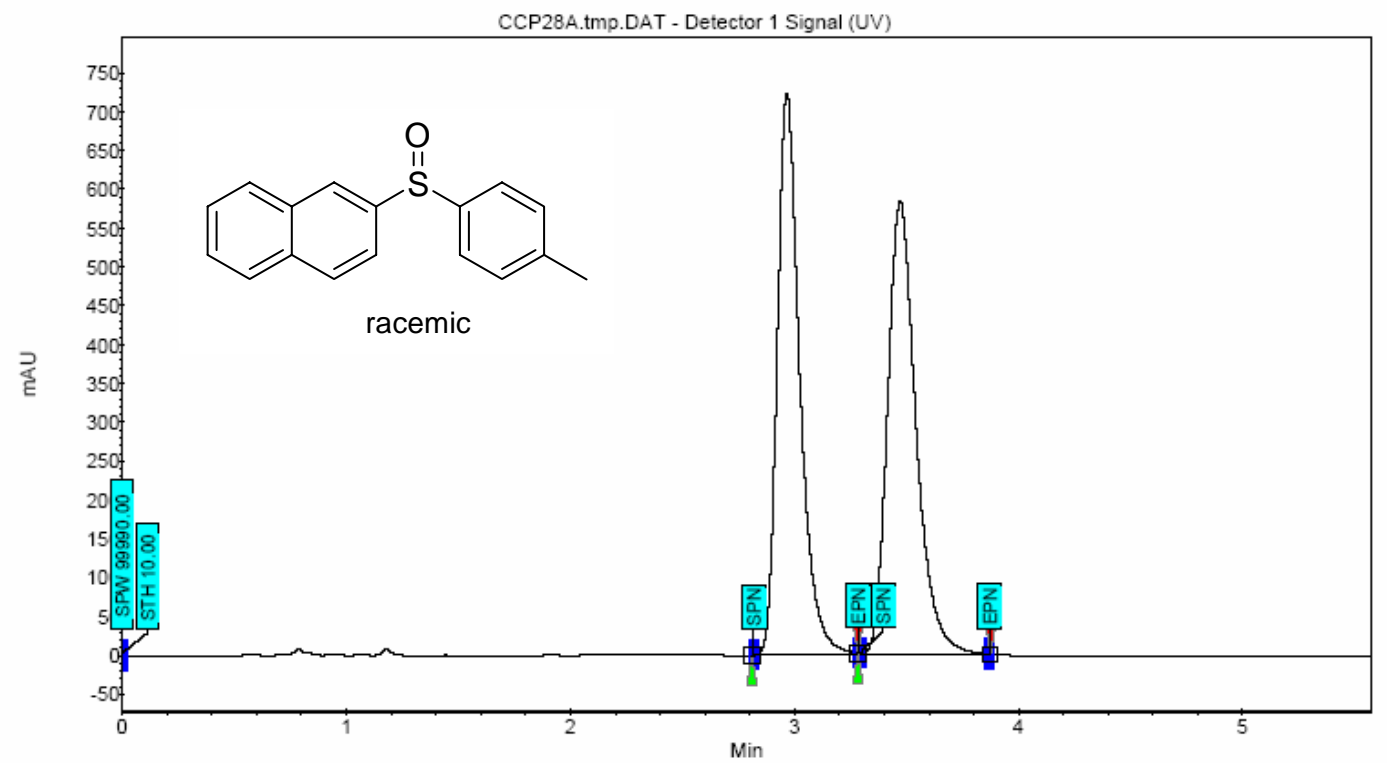

\begin{tabular}{|c|r|r|r|r|r|r|r|}
\hline \multicolumn{1}{|l|}{ Index Name } & \multicolumn{1}{l|}{ Time } & Height & Area & \multicolumn{2}{|c|}{ Area Selectivity Res. HW } \\
\hline \hline & & {$[\mathrm{Min}]$} & {$[\mu \mathrm{V}]$} & {$[\mu \mathrm{\mu} . \mathrm{Min}]$} & {$[\%]$} & & \\
\hline 1 & UNKNOWN & 2.97 & 723.4 & 83.9 & 49.561 & 0.00 & 0.00 \\
\hline 2 & UNKNOWN & 3.47 & 582.4 & 85.4 & 50.439 & 1.17 & 2.53 \\
\hline & & & & & & & \\
\hline Total & & & 1305.8 & 169.3 & 100.000 & & \\
\hline
\end{tabular}

MS34ee0

MS34ee0

ESPCI - Laboratoire de Chimie Organique

sulfoxyde colonne OD-H $5 \mathrm{~m} / \mathrm{min}, 20 \% \mathrm{MeOH} 220 \mathrm{~nm}$

4/6/2007 1:05:56 PM

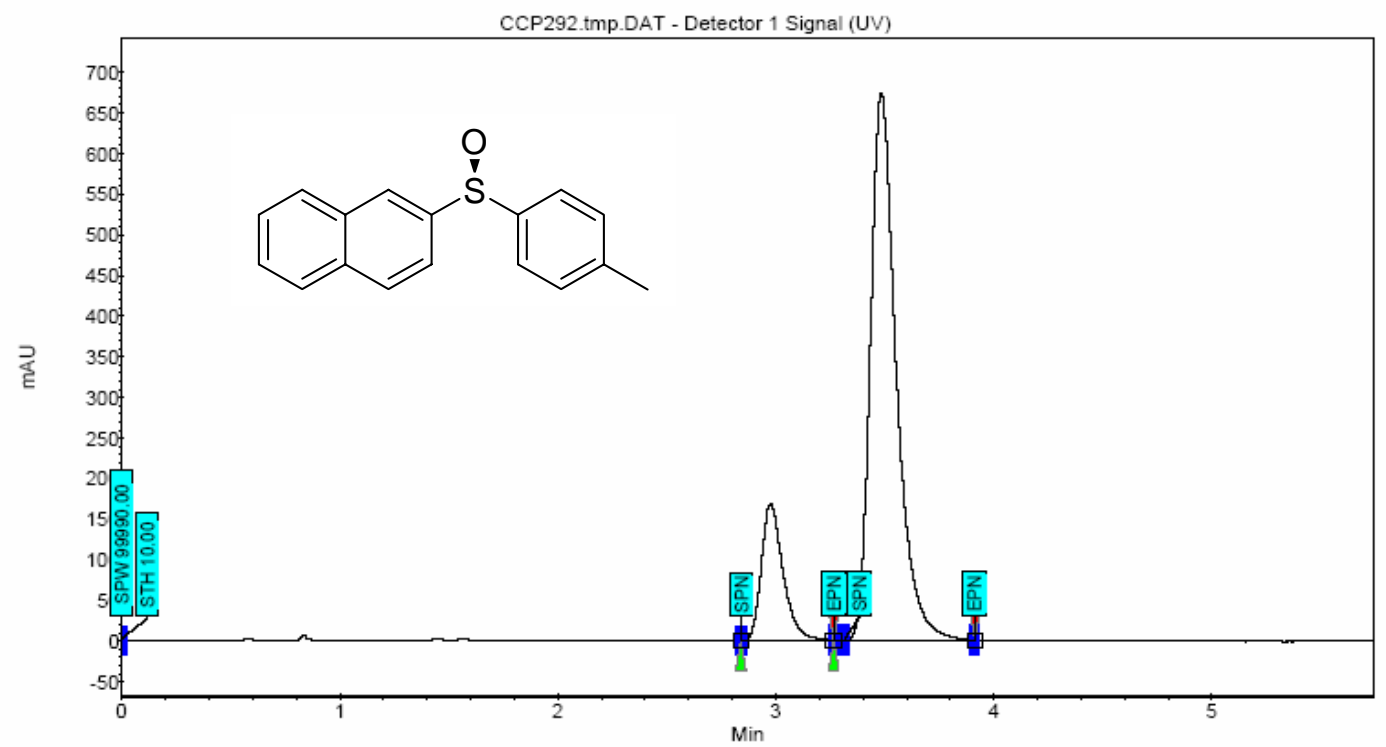

\begin{tabular}{|c|l|r|r|r|r|r|r|}
\hline \multicolumn{2}{|l|}{ Index Name } & \multicolumn{1}{l}{ Time Height } & Area & \multicolumn{2}{|c|}{ Area } & \multicolumn{2}{l|}{ Selectivity Res. HW } \\
\hline \hline & & {$[$ Min] } & {$[\mu \mathrm{V}]$} & {$[\mu \vee . M i n]$} & {$[\%]$} & & \\
\hline 1 & UNKNOWN & 2.98 & 168.7 & 19.4 & 17.331 & 0.00 & 0.00 \\
\hline 2 & UNKNOWN & 3.48 & 674.3 & 92.6 & 82.669 & 1.17 & 2.67 \\
\hline & & & & & & & \\
\hline Total & & & 843.0 & 112.0 & 100.000 & & \\
\hline
\end{tabular}

16 
sulfoxyde colonne OD-H $5 \mathrm{ml} / \mathrm{min}, 5 \% \mathrm{MeOH} 220 \mathrm{~nm}$

4/6/2007 12:41:30 PM

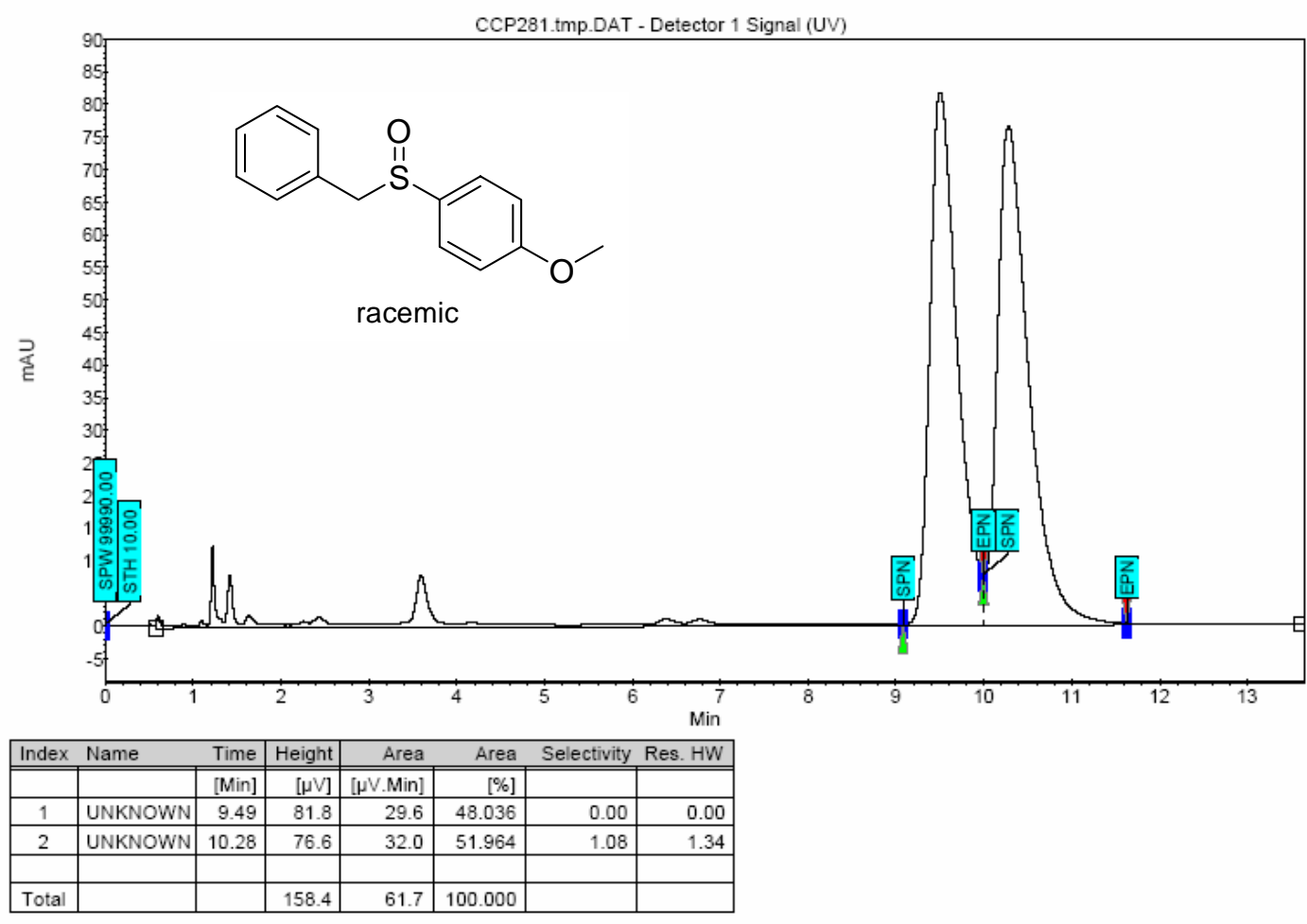

MS27ee0

ESPCI - Laboratoire de Chimie Organique

MS27ee0

sulfoxyde colonne OD-H $5 \mathrm{ml} / \mathrm{min}, 5 \% \mathrm{MeOH} 220 \mathrm{~nm}$

4/6/2007 12:44:38 PM

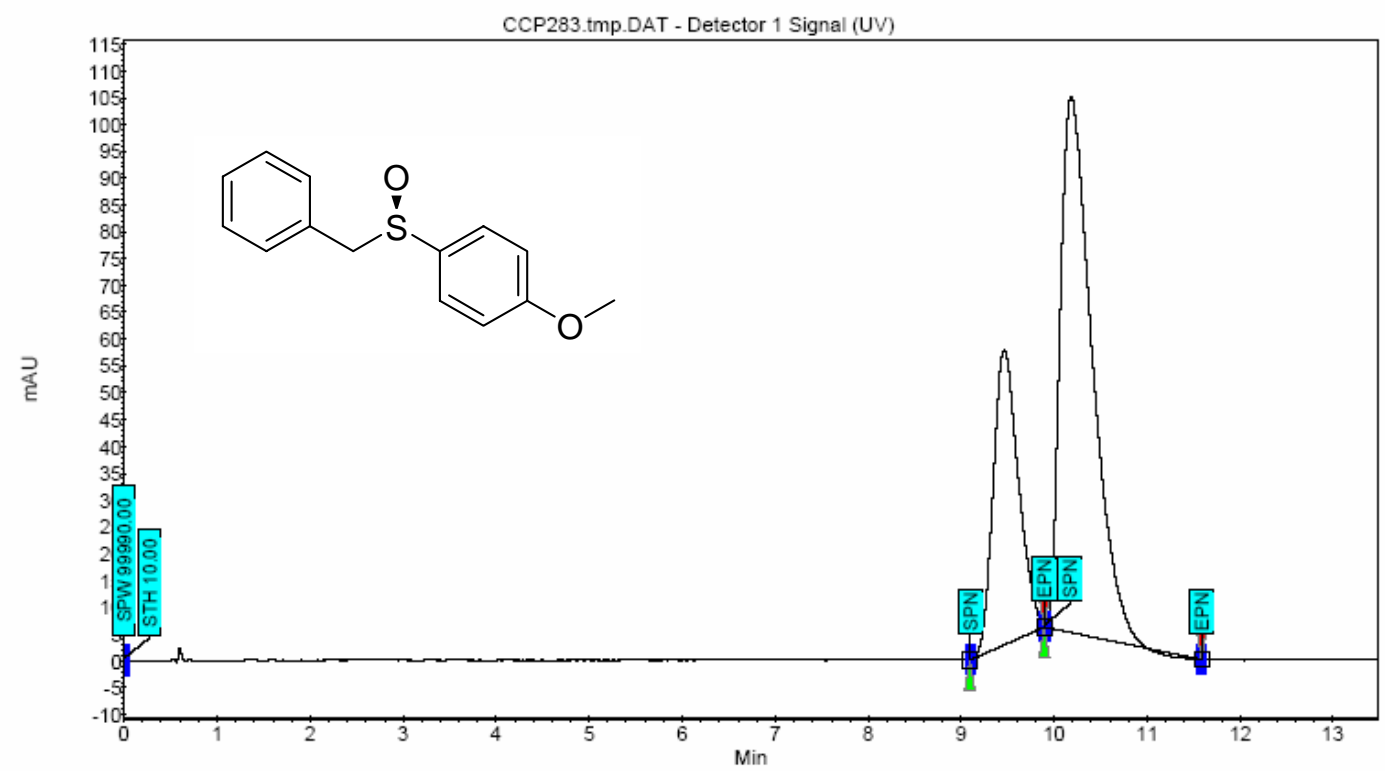

\begin{tabular}{|c|c|c|c|c|c|c|c|}
\hline Index & Name & Time & Height & Area & Area & Selectivity & Res. HW \\
\hline & & [Min] & {$[\mu \mathrm{V}]$} & [ $\mu \vee$ Min] & [\%] & & \\
\hline 1 & UNKNOWN & 9.46 & 54.9 & 17.2 & 30.271 & 0.00 & 0.00 \\
\hline 2 & UNKNOWN & 10.19 & 100.1 & 39.6 & 69.729 & 1.08 & 1.30 \\
\hline & & & & & & & \\
\hline Total & & & 155.1 & 56.8 & 100.000 & & \\
\hline
\end{tabular}


MS32rac0

ESPCI - Laboratoire de Chimie Organique

MS32rac0

sulfoxyde colonne AD-H $5 \mathrm{ml} / \mathrm{min}, 20 \% \mathrm{MeOH} 220 \mathrm{~nm}$

4/6/2007 3:14:15 PM

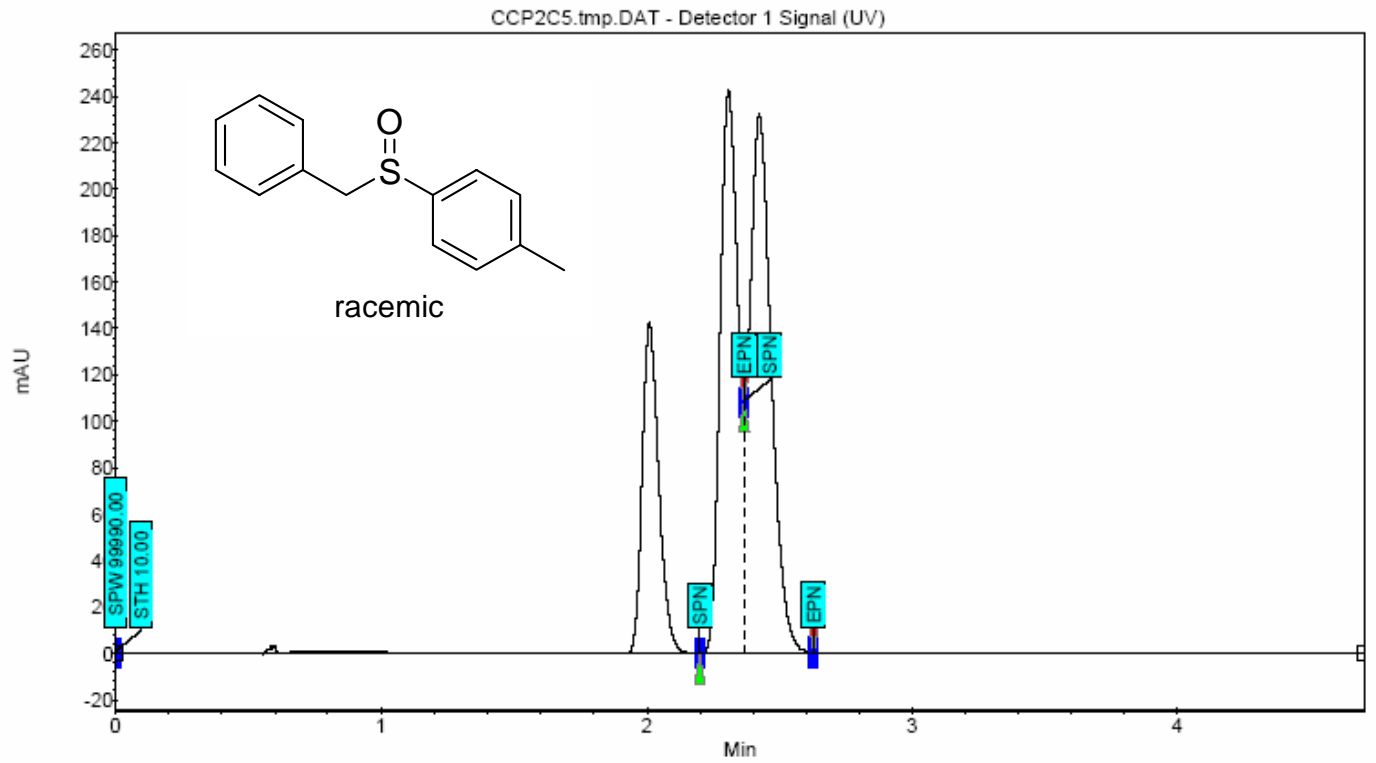

\begin{tabular}{|c|l|r|r|r|r|r|r|}
\hline \multicolumn{1}{|l|}{ Index } & Name & \multicolumn{1}{c|}{ Time } & Height & Area & \multicolumn{2}{|c|}{ Area } & \multicolumn{2}{l|}{ Selectivity Res. HW } \\
\hline \hline & & {$[\mathrm{Min}]$} & {$[\mu \mathrm{V}]$} & {$[\mu \mathrm{V} . \mathrm{Min}]$} & {$[\%]$} & & \\
\hline 1 & UNKNOWN & 2.31 & 243.3 & 18.9 & 45.824 & 0.00 & 0.00 \\
\hline 2 & UNKNOWN & 2.42 & 232.2 & 22.3 & 54.176 & 1.05 & 0.78 \\
\hline & & & & & & & \\
\hline Total & & & 475.5 & 41.2 & 100.000 & & \\
\hline
\end{tabular}

MS32ee0

ESPCI - Laboratoire de Chimie Organique

MS32ee0

sulfoxyde colonne AD-H $5 \mathrm{ml} / \mathrm{min}, 20 \% \mathrm{MeOH} 220 \mathrm{~nm}$

4/6/2007 3:11:37 PM

CCP2C4.tmp.DAT - Detector 1 Signal (UV)

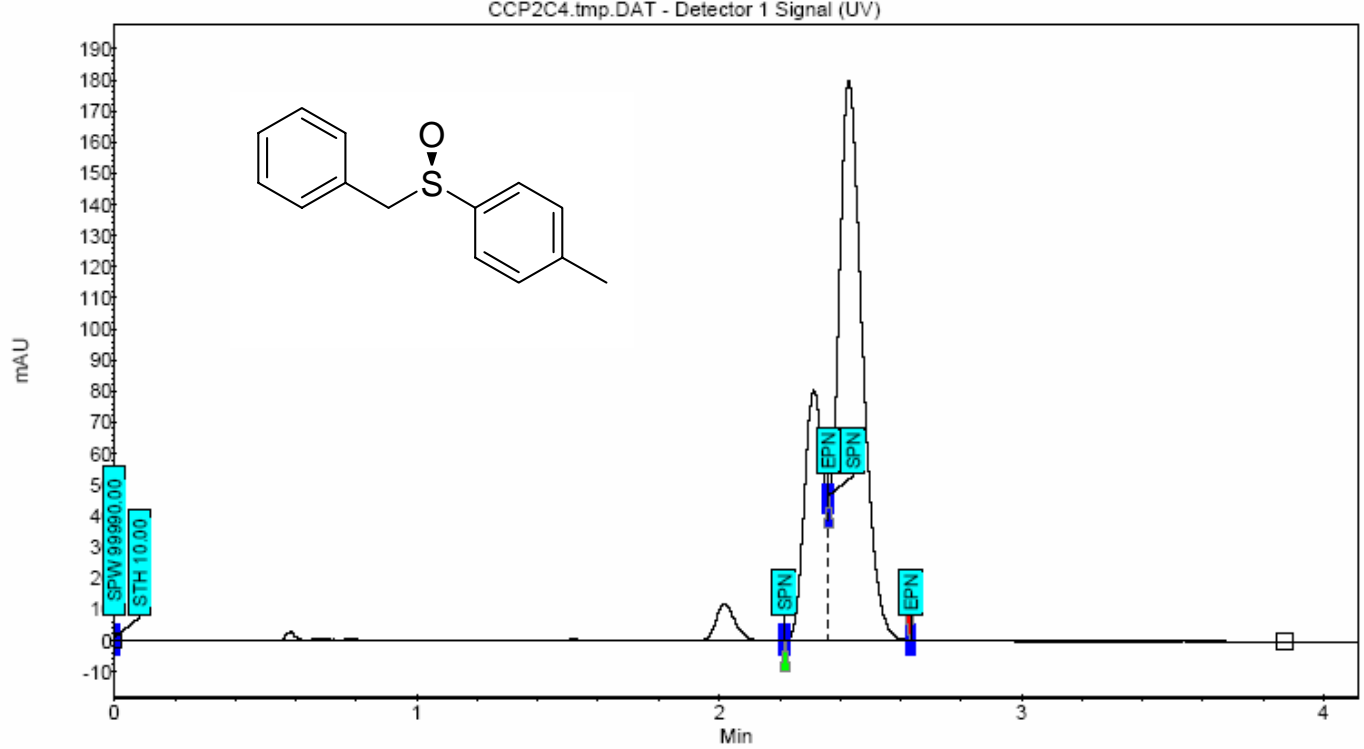

\begin{tabular}{|c|l|r|r|r|r|r|r|}
\hline Index & Name & \multicolumn{1}{l|}{ Time Height } & Area & \multicolumn{2}{|c|}{ Area Selectivity Res. HW } \\
\hline \hline & & {$[\mathrm{Min}]$} & {$[\mu \mathrm{V}]$} & {$[\mu \mathrm{M} . \mathrm{Min}]$} & {$[\%]$} & & \\
\hline 2 & UNKNOWN & 2.31 & 80.5 & 6.0 & 26.223 & 0.00 & 0.00 \\
\hline 1 & UNKNOWN & 2.43 & 180.2 & 16.9 & 73.777 & 1.05 & 0.81 \\
\hline & & & & & & & \\
\hline Total & & & 260.7 & 23.0 & 100.000 & & \\
\hline
\end{tabular}

18 